\title{
Ant-Air Self-learning Algorithm for Path Planning in a Cluttered Environment
}

\author{
Rafiq Ahmad and Peter Plapper
}

\begin{abstract}
Path planning in unstructured area while dealing with narrow spaces is an area of research which is receiving extensive interest. Many existing algorithms are able to produce safe paths but the presented concepts are either not adapted to narrow spaces or they are unable to learn from the past experience to improve repeated movements from the same agent or followed trajectories by other agents. This paper introduces an original concept based on Ant-Air phenomenon for safe path planning in a cluttered environment where narrow passages are treated. The algorithm presented is able to learn from the past experience and hence improve the already generated trajectory further by using some lessons learned from the past experience. The concept is applicable in various domains such as mobile robot path planning, manipulator trajectory generation and part movement in narrow passages in real or virtual assembly/disassembly process.
\end{abstract}

Index Terms-Path planning, collision detection and avoidance, self-learning algorithm, assembling or disassembling, narrow spaces.

\section{INTRODUCTION}

Safe path planning for the accessibility of parts during assembly and disassembly process is a point of interest for many researchers. Usually accessibility verification in (virtual) design process is the safe path calculation for collision free movement of (virtual) part in a (virtual) assembly [1]. Ferre et al. [2] also highlighted the path planning challenges for an object in a complex virtual environment.

Many researchers have been using different techniques to solve path planning problems in various research domains such as robotics [3], [4], CNC machines [5], [6] and assembly/disassembly processes [7]. The C-space approach [8], [9] is one of the widely used method for collision detection, which in its present form works on 2D information from a C-space map but the method proposed stuck in local minima when non-convex obstacles are concerned. A D-plan algorithm [7] is successfully applied to path planning in assembling/disassembling but narrow passages still seem to be an important challenge for many algorithms [7]-[10].

Modern industrial robots are designed for use in assembly process, where they have to pass their robotic arm in a cluttered environment. The same is true for mobile robot path planning in an unknown (industrial) cluttered environment where a robot has to pick and place something from one place

Manuscript received March 19, 2015; revised June 2, 2015. This work was supported by IEE industry and sponsored by FNR-AFR Luxembourg funding.

The authors are with University of Luxembourg, L-1359 Luxembourg, Pakistan (e-mail: rafiq.ahmad@uni.lu, peter.plapper@uni.lu). to another. A successful methods in robotic, $A^{*}$ approach [3], extension of Edsger Dijkstra, determines the cost optimal path from starting position to the target location. The disadvantage of the $\mathrm{A}^{*}$ approach lies in its computation time and less environmental learning capabilities.

Learning from the past experience is important to make the trajectory generation process efficient for multi-agent repeating the same track or single agent offline trajectory that needs improvement. Several past learning method exist in the literature, which can learn from the past collision queries [11] or from the previously developed improved/optimized trajectories [12] and adapt them to a new problem on-hand. Modern path planning methods should work independently from the designer and should be able to adjust their selves to the changing environment and learn from the past mistakes [13] e.g. collisions, changed cluttered environment.

The paper presents an algorithm that can adapt itself to the changed/unknown environment by learning from the past (experienced) trajectories. It helps to develop the structure of the environment for future safe movements in narrow passages which can be applied to many domains discussed above. The algorithm presented in this paper inspired from the Ant-Air phenomenon, will be applicable in areas where multi-agents are working. An agent in this case could be a robot, machine part or any mechanical object desired for safe trajectory. The first agent moving in space discovers the area and keeps record for other agents to follow. This paper introduce agent as "Ant" because of inspiration from ant phenomenon discussed in the next section. Every time a no-go-areas path value (if any) is discovered, a cluster is formed, where the following agents are advised not to go because of a trap to avoid spending much of their time with no productive work. The algorithm contribute to minimize the trajectory time while moving safely in the narrow passages exist in an assembly/disassembly process or robot moving in a cluttered environment.

\section{ANT-AIR CONCEPT}

\section{A. Inspiration}

Ant-Air algorithm presented in this paper is originally inspired from an Ant decision phenomenon when an external air force is applied to it while it is moving on a mission. The Ant-Air concept is the phenomenon of observed change by author in the direction of an Ant in response to an external small air blown onto it while it is moving straight on a flat surface. Suppose an Ant is moving on a plain surface and one blow some air onto it, the Ant changes its direction as long as the impulse air does not blow out the Ant as shown in Fig. 1. 
The impulse air work as an indicator providing some necessary information for the change of planned direction. This concept could be applicable where a change of direction is required. A slight impulse or information to provide intelligence needed when desired a change of direction. When a collision is expected to occur in any path planning scenarios, an impulse air should demand/propose a change in direction. This concept can be generalized into any sort of collision problem where a physical trajectory is sought.

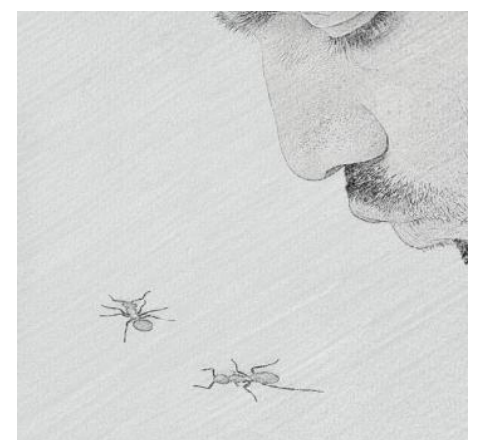

Fig. 1. The concept of "ant-air". (author's observation).

\section{B. Ant-Air Algorithm}

Information from the cluttered scene where an agent "Ant" i.e. a robot or assembly part has to move is taken. This information are acquired in the form of 2D maps, image of the scene or 2D CAD model data. Such information represent a map matrix showing all the information about different objects/obstacles present in the scene. The algorithm need some input trajectory starting point, provisional goal point (optional) to pick up something from and final goal point where the agent Ant has to reach for the final job/delivery.

Once the input information are provided to the algorithm as shown in Fig. 2(a), the algorithm finds a direct short path from the initial point $A$ to the provisional goal point $B$ and then to the final point $\mathrm{C}$. As shown, the algorithm finds a path in a cluttered environment where many obstacles (shown in grey) are present. It therefore detects the potential collisions by checking the obstacle value at the next trajectory point. In the case of collision at the next trajectory point, e.g. while reaching at point A1 in Fig. 2(b), the next point will give a potential collision, the algorithm provides Air-impulse intelligence (alert/warning) to the agent Ant for finding a safe and efficient diversion. At each point, an agent Ant has 8 possibilities and the air impulse provided by the algorithm will force the Agent to change to a feasible direction. At point A1, the proposed concept will search for all the possibilities as shown in Fig. 2(c), step 01. The selection of best direction is done using three criteria:

1) No obstacle present at the selected point;

2) The selected point gives the minimum distance to the next goal point (provisional/final);

3) The point is not already highlighted in buffer cluster i.e. no-go area from past experience.

At point $\mathrm{A} 1$, the point 5 satisfies all the three criteria and therefore is selected as a next trajectory point. Point 5 in (c) is then A2 in (b). From A2, a direct trajectory to provisional point $\mathrm{B}$ is not possible and therefore the same procedure is repeated to select a secure feasible direction as shown in step 02 in (c). Point 7 is selected which becomes A3 in (b) and step 3 in (c), from where a direct movement is possible to the provisional point $\mathrm{B}$. Once a provisional point $\mathrm{B}$ is reached the same process is repeated to reach the final goal point $\mathrm{C}$. Expected collision is detected at point B1 and agent Ant is diverted to the point B2 as detailed in step 4 (c). Finally a safe trajectory is obtained.

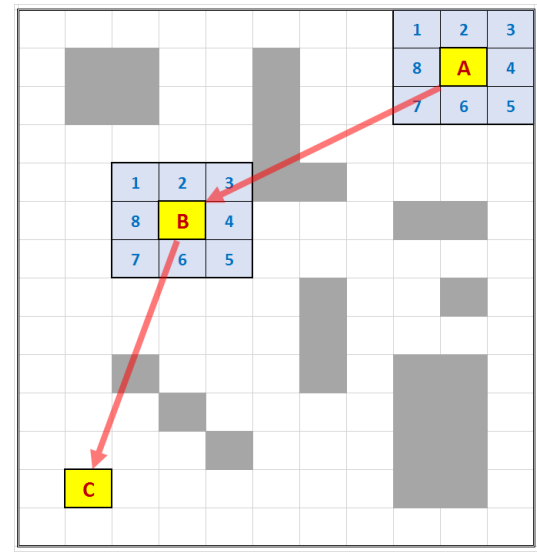

(a)

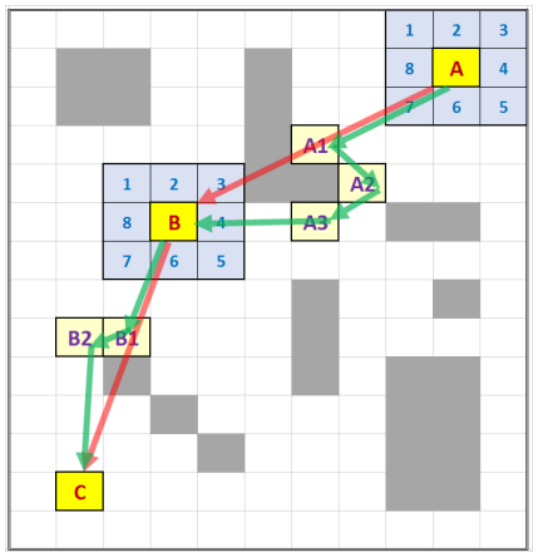

(b)

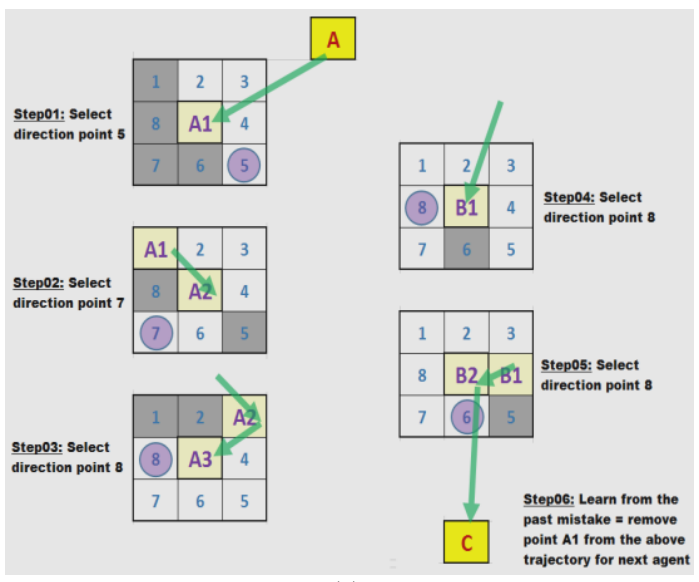

(c)

Fig. 2. Ant-Air algorithm procedure with an imaginary example.

Once a safe trajectory is obtained a buffer cluster of no-go area is defined from experience, where the unnecessary movement from the previous trajectory are learnt and recorded for the next agents to follow. In this concept example discussed, points $\mathrm{A} 1$ and $\mathrm{B} 1$ are determined as unnecessary points and hence no-go areas because without these points the safe trajectory is possible i.e. A-A2-A3-B-B2-C. The buffer concept is much clearly visible in large buffer areas which will be discussed with an example in the result section. The buffer/no-go area obtained 
is stored as a lesson learned and must be avoided unless the scene information matrix are updated and a new exploration is required.

\section{RESULTS}

An agent Ant is moving from the initial point 1 to a provisional point 2 to pick up something that needs to be delivered at the destination point 3 as shown in Fig. 3. Multiple collisions are expected, if a straight line followed between these points. The scene image information and initial trajectory plus goal points are provided to the algorithm developed using Matlab and initial safe trajectory generated for this scenario is shown in Fig. 4. The algorithm find the buffer clusters as shown in Fig. 5 and learns not repeating them. The concept then generates safe trajectory for next working agent or the same agent repeating trajectory as shown in Fig. 6, which is the improved safe trajectory through narrow spaces by using knowledge from the past experience.

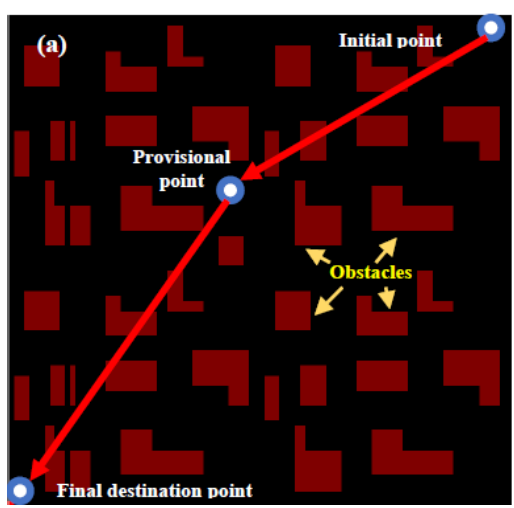

Fig. 3. Initial Trajectory with collisions.

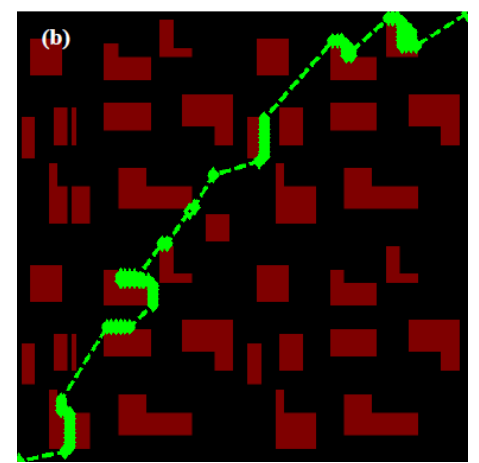

Fig. 4. Results: Initial safe trajectory.

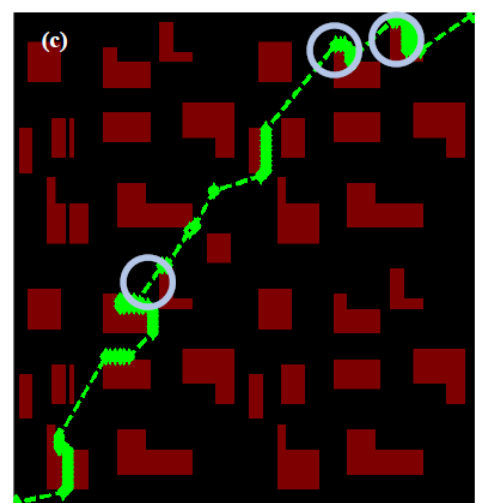

Fig. 5. Results: Buffer cluster formation: learning from past mistakes from initial trajectory as shown in Fig. 4.

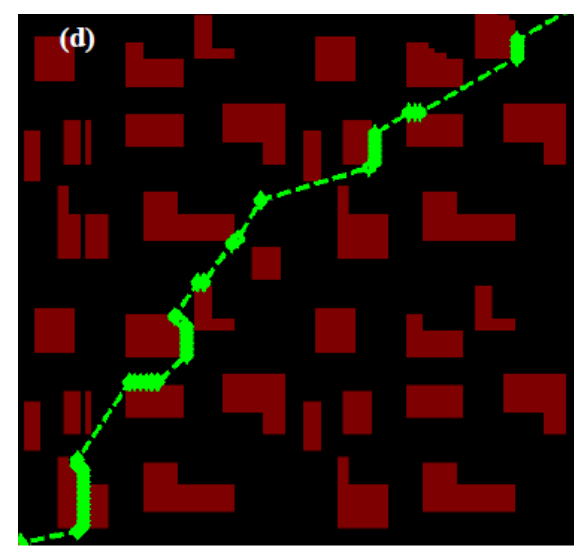

Fig. 6. Results: next iteration (second ant) safe trajectory, after learning from the previous iteration clusters

The limitation of this algorithm lies in its work only for the static environment in its current form. Even though the algorithm is able to find safe path in a dynamic environment but it might not learn from the past experience because the scene information will be changing continuously.

\section{FUTURE APPLICATIONS}

\section{A. Assembling/Disassembling}

Safe path generation in virtual/real assembly and disassembly process is an important area of application for the developed algorithm. Narrow spaces in CAD model will be treated for collision detection and avoidance in future using the developed approach.

\section{B. Robot/Manipulator Path Planning}

Mobile robot path planning is also an important application area for the developed algorithm. A robot should know the original map of the plant in which it is working and should be autonomous to learn from the past to decide which direction should take to avoid collisions and wasting time in the case of buffers (accidents, route blocks, crowd, inventory etc.). An active camera may be used to get information from the scene and safe and efficient trajectory can be calculated for a robot and its following other robots. The same concept can be applied into an industrial robot manipulator path planning in a quasi-static cluttered environment.

\section{CONCLUSION}

Path planning in a cluttered environment is an area of research which needs a generalized approach and learning. The Ant-Air phenomenon based algorithm is presented in this paper, which can detect and avoid collisions in a cluttered environment using obstacles information knowledge from the scene. The concept presented is intelligent (enough) to learn from the previous mistakes and finally improve the next trajectory trials for the next agents to follow. The algorithm finds applications in many path planning domains such as mobile robot moving in a cluttered environment, industrial manipulator working in a cluttered space and virtual or real assembly and disassembly processes.

\section{ACKNOWLEDGMENT}

Authors would like to thank IEE industry for their support 
in providing 3D-MLI sensor and also for their technical support regarding its installation and processing. Authors would also like to thank FNR for funding the research project. Authors would also like to thank Raphael Hinger for his technical help in the demonstration of the results.

\section{REFERENCES}

[1] A. Dugas, J. J. Lee, M. Terrier, and J. Y. Hascoet, "Virtual manufacturing for high speed milling," in Proc. 35th CIRP Intern. Seminar on Manufacturing Systems, South Korea, 2002, pp. 199 - 206.

[2] E. Ferre, J. P. Laumond, G. Arechavaleta, and C. Estevès, "Progresses in assembly path planning," in Proc. International Conference on Product Lifecycle Management, 2005, pp. 373-382.

[3] M. Bennewitz and W. Burgard, "A probabilistic method for planning collision-free trajectories of multiple mobile robots," in Proc. the Applications and Safety Issues in an Emerging Market at the 14th ECAI, 2000

[4] C. W. Warren, "Multiple robot path coordination using artificial potential fields," in Proc. 1990 IEEE International Conference on Robotics and Automation Proceedings, 1990, pp. $500-505$.

[5] R. Ahmad, S. Tichadou, and J. Y. Hascoet, "3D safe and intelligent trajectory generation for multi-axis machine tools using machine vision," International Journal of Computer-Integrated Manufacturing, vol. 26, no. 4, pp. 365-385, 2012.

[6] R. Ahmad, S. Tichadou, and J. Y. Hascoet, "New computer vision based Snakes and Ladders algorithm for the safe trajectory of two axis CNC machines," Journal of Computer Aided Design, vol. 44, no. 5, pp. 355-366, 2011.

[7] L. Zhang, X. Huang, Y. J. Kim, and D. Manocha, "D-plan: Efficient collision-free path computation for part removal and disassembly," Journal of Computer-Aided Design and Applications, 2008.

[8] C. S. Jun, K. Cha, and Y. S. Lee, "Optimizing tool orientations for 5-axis machining by configuration-space search method," Computer-Aided Design, vol. 35, no. 6, pp. 549 - 566, 2003.

[9] Z. Liangjun and D. Manocha, "An efficient retraction-based RRT planner," in Proc. International Conference on Robotics and Automation, pp. 3743-3750, 2008.

[10] J. J. Kuffner, S. M. LaValle, "RRT-connect: An efficient approach to single-query path planning," in Proc. International Conference on Robotics and Automation, pp. 995-1001, 2000.

[11] J. Pan, S. Chitta, D. Manocha, "Faster sample-based motion planning using instance-based learning," Algorithmic Foundations of Robotics X, Springer Berlin Heidelberg, pp. 381-396, 2013.

[12] N. Jetchev and M. Toussaint, "Fast motion planning from experience: trajectory prediction for speeding up movement generation," Auton Robot, pp. 111-127, 2013.

[13] D. Berenson, P. Abeel, and K. Goldberg, "A robot path planning framework that learns from experience," in Proc. IEEE International Conference on Robotics and Automation, pp. 3671-3678, 2012.

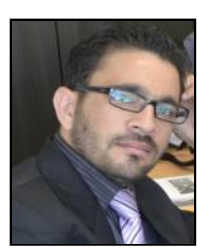

Engr. Rafiq Ahmad was born in Swat, Pakistan in 1983 $\mathrm{He}$ has secured his $\mathrm{PhD}$ in mechanical engineering in September 2012 from IRCCyN, Ecole Cenrale de Nantes, France. His received his master's degree was in knowledge integration in mechanical engineering design and production from Ecole Nationale Supérieure d'Arts et Métiers (ENSAM) Paris, France in 2009. His primary four years bachelor's degree was in mechanical engineering from the University of Engineering and Technology Peshawar, Pakistan in 2007. His major field of study and research includes CNC multi-axis machine tool intelligent manufacturing. Knowledge and vision based planning and decision systems, Industrial automation, Industrial and mobile robots planning systems.

Since Sept 2012 he worked as an assistant professor at CECOS University for a year and then obtained a Luxembourgish FNR funding to work as a senior researcher in the University of Luxembourg. He has also participated in various research and development projects at Ecole Centrale de Nantes, France. He got his first insight into the research and academic world after bachelor's degree where he served as a research assistant at Gulaam Ishaq Khan Institute of Engineering and Technology, Topi, Pakistan.

Dr. Rafiq is a member of ASME, International Institute of Engineers and Pakistan Society of Mechanical Engineers. He is also an active reviewer in various conferences and journals that includes: Computer-aided Design; International Journal of Precision Engineering and Manufacturing; International Journal of Computer Integrated Manufacturing; Chinese Optics Letters Journal; International Journal of Engineering Education; ASME Conference; ICCMA Conference, HSM conference.

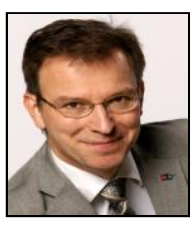

Ing. Peter Plapper was born in Mannheim, Germany in 1963. In 1986 he completed his studies in mechanical engineering / design at TU Kaiserslautern with the degree Dipl.-Ing. His doctoral thesis at the laboratory of tool machines (WZL) of RWTH Aachen, Germany was awarded with the Borchers Medal for scientific excellence in 1993.

Since 1994, he worked for Adam Opel and General Motors in different management positions in Manufacturing Engineering (ME) with increasing responsibility. He developed innovative production technologies, implemented tool machines and coordinated the refurbishment of robotic assembly lines. From 1998 until 2002 he joined the Tech Center of GM in Michigan, USA where he shaped the global manufacturing strategy for Body Shop and General Assembly. During his industrial career he worked on many different robot applications, led the installation of assembly lines all European GM vehicle plants and was responsible as the head of manufacturing engineering for the equipment of all shops in plant Russelsheim. Following his assignment as manager advanced technologies europe Peter Plapper was appointed in 2010 full-professor for manufacturing engineering to the University of Luxembourg.

Prof. Plapper is a member of AIM (European Academy of Industrial Management), VDI (Verein Deutscher Ingenieure), and Luxembourg Materials and Production Cluster Steering Committee. Since 2014 he is the DIRECTOR of the new Master program "Master of Science in Engineering - Sustainable Product Creation". For the current list of publications please visit . 